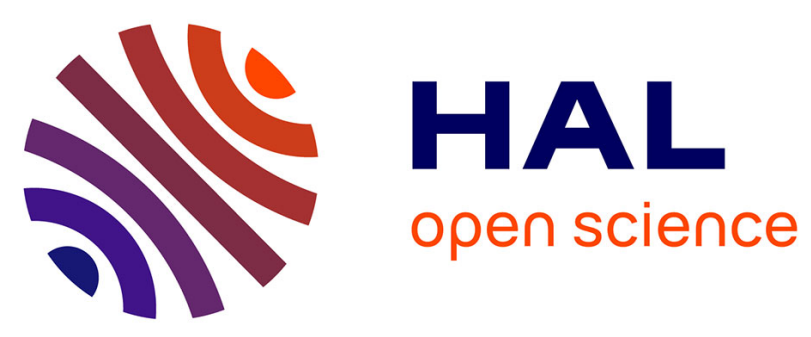

\title{
Stone-Weierstrass Theorem
}

Guy Laville, Ivan Ramadanoff

\section{- To cite this version:}

| Guy Laville, Ivan Ramadanoff. Stone-Weierstrass Theorem. 2007. hal-00003218v2

\section{HAL Id: hal-00003218 \\ https://hal.science/hal-00003218v2}

Preprint submitted on 25 Jan 2007

HAL is a multi-disciplinary open access archive for the deposit and dissemination of scientific research documents, whether they are published or not. The documents may come from teaching and research institutions in France or abroad, or from public or private research centers.
L'archive ouverte pluridisciplinaire HAL, est destinée au dépôt et à la diffusion de documents scientifiques de niveau recherche, publiés ou non, émanant des établissements d'enseignement et de recherche français ou étrangers, des laboratoires publics ou privés. 


\title{
STONE-WEIERSTRASS THEOREM \\ G. LAVILLE and I.P. RAMADANOFF
}

\begin{abstract}
It will be shown that the Stone-Weierstrass theorem for Cliffordvalued functions is true for the case of even dimension. It remains valid for the odd dimension if we add a stability condition by principal automorphism.
\end{abstract}

Introduction.- Recall the classical Stone-Weierstrass theorem : let $Y$ be a metric space, $\mathcal{C}(Y ; \mathbb{R})$ the set of all continuous functions from $Y$ in $\mathbb{R}$, $B \subset \mathcal{C}(Y ; \mathbb{R})$ a subset such that $B$ contains the constant function 1 and separates the points of $Y$. Then the algebra $A_{B}(Y ; \mathbb{R})$, generated by $B$ is dense in $\mathcal{C}(Y ; \mathbb{R})$ for the topology of the uniform convergence on every compact.

It is well-known that if one substitutes the field $\mathbb{R}$ by $\mathbb{C}$, then an additional hypothesis is needed, namely : $B$ should be stable with respect to complex conjugation. In case we are omitting this hypothesis and if we take, for example, $Y$ to be an open subset of $\mathbb{C}$ and $Y=\{1, z\}$, then we will get the algebra of holomorphic functions.

Let us mention that the case of functions taking values in the quaternonian field is known [2] and it is analogous to the real case.

Here, we will investigate the situation when $\mathbb{R}$ is replaced by $\mathbb{R}_{p, q}$ - an universal Clifford algebra of $\mathbb{R}^{n}, n=p+q$, with a quadratic form of signature $(p, q)$. This study is motivated by the theory of monogenic functions [1]. The present paper is organized as follows : in the $\oint 1$ we will recall some notations usually employed in Clifford algebras. The $\S 2$ will deal with some elements of combinatorics. The essential part of the paper in the $\S 3$ in which we give a formula allowing to compute the scalar part of a given Clifford number. As an application of this formula, we are able to prove in $\S 4$ the following Stone-Weierstrass theorem for $\mathcal{C}\left(Y ; \mathbb{R}_{p, q}\right)$ :

Theorem.- Let $Y$ be a metric space and $\mathcal{C}\left(Y ; \mathbb{R}_{p, q}\right)$ the set of all continuous functions from $Y$ to $\mathbb{R}_{p, q}$. Let $B \subset \mathcal{C}\left(Y, \mathbb{R}_{p, q}\right)$ be such that $B$ contains the constant function 1 and separates the points of $Y$. If $p+q$ is odd, suppose in addition that $B$ is stable with respect to the principal automorphism *. Then, the algebra $A_{B}\left(Y ; \mathbb{R}_{p, q}\right)$, generated by $B$, is dense in $\mathcal{C}\left(Y ; \mathbb{R}_{p, q}\right)$ for the topology of uniform convergence on compact sets. 


\section{Notations}

In a Clifford algebra $\mathbb{R}_{p, q}=C_{0} \oplus C_{1} \oplus \ldots \oplus C_{n}$, with $n=p+q$, the spaces $C_{0}, C_{1}, \ldots, C_{n}$ are supposed to be of respective basis $\{1\},\left\{e_{1}, e_{2}, \ldots, e_{n}\right\}$, $\left\{e_{i j}\right\}_{i<j}, \ldots,\left\{e_{i_{1} \ldots i_{k}}\right\}_{i_{1}<i_{2}<\cdots<i_{k}}, \ldots,\left\{e_{1.2 \ldots n}\right\}$, where $\left(i_{1}, \ldots, i_{k}\right)$ is a multiindex with $i_{1}, \ldots, i_{k} \in\{1, \ldots, n\}, 1 \leq i_{1}<\ldots<i_{k} \leq n$. The algebra obeys to the laws :

$$
\begin{cases}e_{i}^{2}=1, & i=1, \ldots, p \\ e_{i}^{2}=-1, & i=p+1, \ldots, n \\ e_{i} e_{j}=-e_{j} e_{i}, & i \neq j \\ e_{i_{1} \ldots i_{k}}=e_{i_{1}} e_{i_{2}} \cdots e_{i_{k}}, & \text { for } i_{1}<i_{2}<\ldots<i_{k}\end{cases}
$$

We will make use of the decomposition of a Clifford number a in its scalar (real) part $\langle a\rangle_{0}$, its 1 -vector $\langle a\rangle_{1} \in C_{1}$, its bivector part $\langle a\rangle_{2} \in C_{2}$, etc ... up to its pseudo-scalar part $\langle a\rangle_{n} \in C_{n}$, i.e :

$$
a=\langle a\rangle_{0}+\langle a\rangle_{1}+\cdots+\langle a\rangle_{n}
$$

where :

$$
\langle a\rangle_{k}=\sum_{\substack{J \\|J|=k}} a_{J} e_{J} .
$$

Where $J=\left(j_{1}, \ldots, j_{k}\right)$ is a multiindice and $|J|=k, \quad e_{J}=e_{j_{1}} \cdots e_{j_{k}}$.

Recall that the principal involution ${ }_{*}$, the anti-involution ${ }^{*}$ and the reversion $\sim$ act on $a \in \mathbb{R}_{0, n}$ as follows :

$$
\begin{aligned}
& a_{*}=\sum_{k=0}^{n}(-1)^{k}\langle a\rangle_{k} \\
& a^{*}=\sum_{k=0}^{n}(-1)^{\frac{k(k+1)}{2}}\langle a\rangle_{k} \\
& a^{\sim}=\sum_{k=0}^{n}(-1)^{\frac{k(k-1)}{2}}\langle a\rangle_{k}
\end{aligned}
$$

Now, define

$$
e^{i}=\left\{\begin{array}{lll}
e_{i}, & \text { if } \quad 1 \leq i \leq p \\
-e_{i}, & \text { if } \quad p+1 \leq i \leq p+q
\end{array}\right.
$$

and $e^{J}=e^{j_{k}} \cdots e^{j_{1}}$. 


\section{Some combinatorics}

Let us study the partition of the set $\{1, \ldots, n\}$ in two strictly ordered subsets: $I=\left\{i_{1}, \ldots, i_{k}\right\}$ and $J=\left\{j_{1}, \ldots, j_{p}\right\}$. As for as the relative position of $J$ with respect to $I$ is concerned, we have different possible cases : $J \cap I=\phi$; just one $j_{\alpha}$ belongs to $I ; \ldots ; \ell$ among the $j_{\alpha}^{\prime} s$ belong to $I ; \ldots$; the largest possible number of $j_{\alpha}^{\prime} s$ belongs to $I$. It is easy to compute the cardinals of the corresponding sets :

For the first case, the cardinal is $C_{n-k}^{p} C_{k}^{\sup \{0, p-(n-k)\}}$. If just one $j_{\alpha}$ belongs to $I$, then we will have $C_{n-k}^{p-1} C_{k}^{\sup \{0, p-(n-k)\}+1}$ and so on ... In the last case, we will get $C_{n-k}^{0} C_{k}^{\inf \{p, k\}}$.

Now, recall the following result which is well-known in classical probability theorey [3] :

Lemma 1.- For every $k, 0 \leq k \leq n$ :

$$
\sum_{\ell=\sup \{0, p-(n-k)\}}^{\inf \{p, k\}} C_{n-k}^{p-\ell} C_{k}^{\ell}=C_{n}^{p} .
$$

In fact, this lemma will not be used here, but its elementary proof, which will be given below, is a source of inspiration for the next result (Lemma 2).

Proof of Lemma 1 - For every $k, \quad 0 \leq k \leq n$, one has $(1+x)^{n-k}(1+x)^{k}=(1+x)^{n}$, which involves

$\sum_{\ell=0}^{k}(1+x)^{n-k} C_{k}^{\ell} x^{\ell}=\sum_{p=0}^{n} C_{n}^{p} x^{p}$, and again :

$\sum_{\ell=0}^{k} \sum_{n=0}^{n-k} C_{n-k}^{n} x^{n} \quad C_{k}^{\ell} x^{\ell}=\sum_{p=0}^{n} C_{n}^{p} x^{p}$. Let us set $n+\ell=p$, i.e. $n=p-\ell$.

Then the double sum is equal to

$$
\sum_{\ell=0}^{k} \sum_{p=\ell}^{n-k+\ell} C_{n-k}^{p-\ell} C_{k}^{\ell} x^{p}=\sum_{p=0}^{n} \sum_{\ell=\sup \{0, p-(n-k)\}}^{\inf \{p, k\}} C_{n-k}^{p-\ell} C_{k}^{\ell} x^{p} .
$$

It just remains to indentify the coefficients of $x^{p}$. Now, we are in a position to formulate and prove the following :

\section{Lemma 2.-}

$$
\sum_{p=0}^{n} \sum_{\ell=\sup \{0, p-(n-k)\}}^{\inf \{p, k\}}(-1)^{p k+\ell} C_{n-k}^{p-\ell} C_{k}^{\ell}= \begin{cases}0, & \text { if } 1 \leq k \leq n-1 \\ 0, & \text { if } k=n, n \text { even } \\ 2^{n}, & \text { if } k=n, n \text { odd } \\ 2^{n}, & \text { if } k=0 .\end{cases}
$$


Proof of Lemma 2 - Start from $\left(1+(-1)^{k} x\right)^{n-k}\left(1+(-1)^{k+1} x\right)^{k}=$

$$
\begin{aligned}
& =\sum_{\ell=0}^{k}\left(1+(-1)^{k} x\right)^{n-k}(-1)^{(k+1) \ell} C_{k}^{\ell} x^{\ell}= \\
& =\sum_{\ell=0}^{k} \sum_{n=0}^{n-k}(-1)^{k n} C_{n-k}^{n} x^{n}(-1)^{(k+1) \ell} C_{k}^{\ell} x^{\ell}= \\
& =\sum_{p=0}^{n} \sum_{\ell=\sup \{0, p-(n-k)\}}^{\inf \{p, k\}}(-1)^{p k+\ell} C_{n-k}^{p-\ell} C_{k}^{\ell} x^{p},
\end{aligned}
$$

because $k n+(k+1) \ell=p k+\ell$. Thus it is enough to set $x=1$ and remark that :

$$
\left(1+(-1)^{k}\right)^{n-k}\left(1+(-1)^{k+1}\right)^{k}= \begin{cases}2^{n}, & \text { if } k=0 \\ 0, & \text { if } 1 \leq k \leq n-1 \\ 2^{n}, & \text { if } k=n, n \text { odd } \\ 0, & \text { if } k=n, n \text { even }\end{cases}
$$

\section{A formula for the real part of $a \in \mathrm{R}_{p, q}$}

Lemma 3.- For every multiindice $J$, we have $e_{J} e^{J}=1$.

Lemma 4.- Let $I=\left(i_{1}, \ldots, i_{k}\right), \quad|I|=k . \quad J=\left(j_{1}, \ldots, j_{p}\right), \quad|J|=p$ there is the following equality

$$
\sum_{p=0}^{n} \sum_{|J|=p} e_{J} e_{I} e^{J}= \begin{cases}2^{n} & \text { if } k=0 \text { or if } k=n \text { with } n \text { odd } \\ 0 & \text { in other cases }\end{cases}
$$

Proof - Decompose the sum

$$
\sum_{|J|=p} e_{j} e_{I} e^{J}
$$

following the relative position of $J$ with respect to $I$. If $J \cap I=\phi$ we have $C_{n-k}^{p} C_{k}^{0}$ such possibilities and the anticommutation gives $(-1)^{p k}$.

If only one $j_{\alpha} \in I$ we have $C_{n-k}^{p-1} C_{k}^{1}$ such possibilites and the anticommutation gives $(-1)^{(p-1) k}(-1)^{k-1}$ and so on, $\ldots$, if $\ell j_{\alpha} \in I$ we have $C_{n-k}^{(p-\ell) k} C_{k}^{\ell}$ such possibilities and the commutation gives $(-1)^{(p-\ell) k}(-1)^{\ell(k-1)}$. 
The sum is equal to

$$
\sum_{\ell=\sup \{0, p-(n-k)\}}^{\inf \{p, k)}(-1)^{(p-\ell) k}(-1)^{\ell(k-1)} C_{n-k}^{p-\ell} C_{k}^{\ell} e_{I}
$$

Thus we could apply lemma 2 and the result follows.

The next result is a formula for the scalar part of a Clifford number.

Theorem 1.- Let $a \in \mathbb{R}_{p, q}$. Then :

a) if $n$ is even,

$$
\langle a\rangle_{0}=\frac{1}{2^{n}} \sum_{p=0}^{n} \sum_{|J|=p} e_{J} a e^{J}
$$

b) if $n$ is odd,

$$
\langle a\rangle_{0}=\frac{1}{2^{n+1}} \sum_{p=0}^{n} \sum_{|J|=p} e_{J} a e^{J}+\frac{1}{2^{n+1}} \sum_{p=0}^{n} \sum_{|J|=p} e_{J} a_{*} e^{J} .
$$

Proof - When $a \in \mathbb{R}_{0, n}$, then

$$
a=\sum_{k=0}^{n} \sum_{|I|=k} a_{I} e_{I}
$$

where $I=\left(i_{1}, \ldots, i_{k}\right), \quad 1 \leq i_{1}<i_{2}<\ldots<i_{k} \leq n$. Take the sum

$$
\sum_{p=0}^{n} \sum_{|J|=p} e_{J} a e^{J}=\sum_{J} \sum_{I} a_{I} e_{J} e_{I} e^{J}
$$

Now, apply lemma 4 :

a) if $n$ is even, one gets :

$$
\sum_{p=0}^{n} \sum_{|J|=p} e_{J} a e^{J}=2^{n}\langle a\rangle_{0}
$$

b) if $n$ is odd, one has :

$$
\sum_{p=0}^{n} \sum_{|J|=p} e_{J} a e^{J}=2^{n}\langle a\rangle_{0}+2^{n}\langle a\rangle_{n}
$$


But, in the case when $n$ is odd, $\left\langle a_{*}\right\rangle_{n}=(-1)^{n}\langle a\rangle_{n}=-\langle a\rangle_{n}$. Thus, we get the part b) of the theorem.

Remark.- For $n=1$, the preceding formula becomes to $4 R e a=(a-i a i)+(\bar{a}-i \bar{a} i)$ in $\mathbb{R}_{0,1}=\mathbb{C}$ with the classical notations of $\mathbb{C}$.

For $n=2$, this means that 4 Re $a=a-i a i-j a j-k a k$ in $\mathbb{R}_{0,2}=\mathbb{H}$ with the classical notations of $\mathbb{H},[2]$.

\section{The Stone-Weierstrass theorem for $\mathcal{C}\left(Y ; \mathrm{IR}_{p, q}\right)$.}

Theorem 3.- Let $Y$ be a metric space and $\mathcal{C}\left(Y ; \mathbb{R}_{p, q}\right)$ the set of continuous functions from $Y$ into $\mathbb{R}_{p, q}$. Let $B \subset \mathcal{C}\left(Y ; \mathbb{R}_{p, q}\right)$ be such that $B$ contains the constant function 1 and separates the points of $Y$. When $p+q$ is even, nothing more is supposed. If $p+q$ is odd, suppose $B$ be stable with respect to the principal involution $*$.

Then, the algebra $A_{B}\left(Y ; \mathbb{R}_{p, q}\right)$, generated by $B$, is dense in $\mathcal{C}\left(Y ; \mathbb{R}_{p, q}\right)$ for the topology of uniform convergence on compact.

Proof - Set $A_{B}(Y ; \mathbb{R})$ for the subspace of $A_{B}\left(Y ; \mathbb{R}_{p, q}\right)$ consisting of those functions which take real values. This is a real algebra. Let $A_{B}(Y ; \mathbb{R})_{I}$ be the subspace of $A_{B}\left(Y ; \mathbb{R}_{p, q}\right)$ consisting of the $I$-components of functions from $A_{B}\left(Y ; \mathbb{R}_{p, q}\right)$. Thus, we have $f_{I}=\left\langle f e^{I}\right\rangle_{0}$ and $A_{B}(Y ; \mathbb{R})_{I} \subset A_{B}(Y ; \mathbb{R})$ by theorem 2 .

In this way, $A_{B}(Y ; \mathbb{R})$ satisfies to the hypothesis of the classical StoneWeierstrass theorem for real functions. The algebra $A_{B}(Y ; \mathbb{R})$ is consequently dense in $\mathcal{C}(Y ; \mathbb{R})$. Finally, one can conclude that :

$$
A_{B}\left(Y ; \mathbb{R}_{p, q}\right)=\bigoplus_{I} A_{B}(Y ; \mathbb{R}) e_{I}
$$

is dense in $\mathcal{C}\left(Y ; \mathbb{R}_{p, q}\right)$.

\section{A remark}

It should be noted that the computations of the scalar part it strongly related to formulas related to the Hestenes multivector derivative : see [4], chapter 2 . 


\section{References :}

[1] R. DELANGHE, F. SOMMEN, V. SANCĚK - Clifford Algebra and Spinor-valued functions ; Kluwer.

[2] J. DUGUNDJI - Topology ; Allyn and Bacon .

[3] W. FELLER - An introduction to the theory of Probability and its applications ; J. Wiley.

[4] D. HESTENES, G. SOBCZYK - Clifford Algebra to Geometric Calculus ; Reidel.

Université de Caen Département de Mathématiques Esplanade de la Paix 14032 CAEN CEDEX - FRANCE 